\title{
Effect of glucose on poly- $\gamma$-glutamic acid metabolism in Bacillus licheniformis
}

\author{
Wencheng Yu ${ }^{1,2+}$, Zhen Chen ${ }^{1,2+}$, Hong Ye ${ }^{1,2}$, Peize Liu ${ }^{1,2}$, Zhipeng Li ${ }^{3}$, Yuanpeng Wang ${ }^{1,2}$, Qingbiao Li ${ }^{1,2}$,
} Shan Yan ${ }^{4}$, Chuan-jian Zhong ${ }^{4}$ and Ning $\mathrm{He}^{1,2^{*}}$

\begin{abstract}
Background: Poly-gamma-glutamic acid ( $\gamma-P G A)$ is a promising macromolecule with potential as a replacement for chemosynthetic polymers. $Y$-PGA can be produced by many microorganisms, including Bacillus species. Bacillus licheniformis CGMCC2876 secretes Y-PGA when using glycerol and trisodium citrate as its optimal carbon sources and secretes polysaccharides when using glucose as the sole carbon source. To better understand the metabolic mechanism underlying the secretion of polymeric substances, SWATH was applied to investigate the effect of glucose on the production of polysaccharides and $\mathrm{Y}-\mathrm{PGA}$ at the proteome level.

Results: The addition of glucose at 5 or $10 \mathrm{~g} / \mathrm{L}$ of glucose decreased the $\mathrm{\gamma}$-PGA concentration by 31.54 or $61.62 \%$, respectively, whereas the polysaccharide concentration increased from 5.2 to $43.47 \%$. Several proteins playing related roles in $\gamma-P G A$ and polysaccharide synthesis were identified using the SWATH acquisition LC-MS/MS method. CcpA and $C c p N$ co-enhanced glycolysis and suppressed carbon flux into the TCA cycle, consequently slowing glutamic acid synthesis. On the other hand, $C c p N$ cut off the carbon flux from glycerol metabolism and further reduced $\gamma$-PGA production. CcpA activated a series of operons ( $g / m$ and epsA-O) to reallocate the carbon flux to polysaccharide synthesis when glucose was present. The production of $\mathrm{Y}$-PGA was influenced by $\mathrm{NrgB}$, which converted the major nitrogen metabolic flux between $\mathrm{NH}_{4}{ }^{+}$and glutamate.
\end{abstract}

Conclusion: The mechanism by which B. licheniformis regulates two macromolecules was proposed for the first time in this paper. This genetic information will facilitate the engineering of bacteria for practicable strategies for the fermentation of Y-PGA and polysaccharides for diverse applications.

Keywords: Glucose, Y-PGA, Polysaccharide, Carbon control protein, B. licheniformis

\section{Background}

Poly-gamma-glutamic acid ( $\gamma$-PGA) is a type of polyamide composed of single glutamic acids joined via $\gamma$-amide linkages between the glutamate $\gamma$-carboxyl and $\alpha$-amino groups [1]. $\gamma$-PGA is a natural macromolecular polymer that is biodegradable, edible and non-toxic. Thus, $\gamma$-PGA and its derivatives have been applied in diverse fields, particularly as flocculants in water treatment and algal collection [2-4].

\footnotetext{
*Correspondence: hening@xmu.edu.cn.

'Wencheng Yu and Zhen Chen contributed equally to this work

${ }^{1}$ Department of Chemical and Biochemical Engineering, College

of Chemistry and Chemical Engineering, Xiamen University,

Xiamen 361005, People's Republic of China

Full list of author information is available at the end of the article
}

The selection of carbon sources for $\gamma$-PGA production is strain-dependent. Glucose and glycerol were both reported to favor $\gamma$-PGA production in most strains [5]. Importantly, glucose plays different roles in different $\gamma$-PGA-producing strains. Bacillus licheniformis ATCC9945a converts glucose to $\alpha$-ketoglutarate via glycolysis and the TCA cycle, followed by the production of glutamic acid to synthesize $\gamma$-PGA. Moreover, glucose is reported to be a better carbon source than glycerol for the growth of B. licheniformis ATCC9945a [6]. For Bacillus subtilis NX-2, glucose is primarily utilized as an energy source for cell growth during $\gamma$-PGA biosynthesis, whereas glutamate in the medium is the main precursor for $\gamma$-PGA formation [7]. In contrast, using glycerol as the sole carbon source, Bacillus amyloliquefaciens 
C06 produces $\gamma$-PGA containing polysaccharides as byproducts [8]. Some genetic information regarding the effect of glucose on $\gamma$-PGA synthesis has been reported. Msadek et al. demonstrated that the presence of glucose in the medium resulted in a decline in $\gamma$-PGA production because glucose suppressed the transcription of $\operatorname{deg} Q$, which activated the $\operatorname{Cap} A B C$ operon $[9,10]$.

Metabolic engineering has sought to improve $\gamma$-PGA production. In a $B$. amyloliquefaciens M306 mutant obtained by Liu et al. the $\gamma$-PGA yield increased from 3.2 to $6.8 \mathrm{~g} / \mathrm{L}$ through the down-regulation of epsD and $y q x M$ expression [11]. A study from Feng et al. demonstrated that the epsA-O deletion in B. amyloliquefaciens NK-1 contributed to a significant improvement in $\gamma$-PGA production $(5.12 \mathrm{~g} / \mathrm{L})$, which represented a $63.2 \%$ increase compared to the wild-type strain; moreover, the $\gamma$-PGA purity improved from 76.8 to $80.4 \%$ [12]. These results indicate the existence of an unknown competition mechanism between the synthesis of $\gamma$-PGA and polysaccharides. Thus, the metabolic regulation system in these strains may control the synthesis of both extracellular polymeric substances in response to environmental changes.

In our previous studies, B. licheniformis CGMCC2876 was observed to produce extracellular polysaccharides when using glucose as the sole carbon source [13, 14], whereas poly- $\gamma$-glutamic acid ( $\gamma$-PGA) was secreted when trisodium citrate and glycerol were used as the carbon sources [15]. Both of the extracellular polymeric substances exhibited high flocculating activities. Polymers with different components and molecular weights are required for different purposes, and controlling the components and molecular weights has fundamental and practical importance for commercial development $[16,17]$. To better understand the metabolic mechanism underlying the section of extracellular polymeric substances, we investigated the effect of glucose on the production of polysaccharides and $\gamma$-PGA at the proteome level. Sequential window acquisition of all theoretical fragment-ion spectra (SWATH) acquisition LC-MS/MS was used to analyze the differentially expressed proteins in B. licheniformis cultured under different conditions. Finally, we proposed a mechanism for regulating the metabolism of these two macromolecules in B. licheniformis.

\section{Results and discussion}

Bacillus licheniformis cell growth in culture media with different glucose concentrations

Previous studies showed that glucose and citric acid were the better carbon sources for most $\gamma$-PGA producing strains $[6,18,19]$. Our result in Fig. 1 showed that the lag time of cell growth was significantly shortened when glucose was added and that the maximum biomass was $30 \%$ less than the biomass in the $\gamma$-PGA medium. Carbon source and $\mathrm{C} / \mathrm{N}$ ratio are important factors for bacterial growth and accumulation of secondary metabolites [16]. An abundant carbon source can accelerate microbial growth. However, overabundance of carbon sources is not conducive to bacterial reproduction [20]. Additionally, glycerol was utilized after glucose was exhausted. A similar result was previously reported for B. licheniformis ATCC9945a, suggesting that glycerol utilization is suppressed in medium containing the glucose/glycerol mixture [6].

\section{Effect of glucose on B. licheniformis fermentation products}

As shown in Table 1, $8.45 \mathrm{~g} / \mathrm{L}$ polysaccharide was produced in medium containing $10 \mathrm{~g} / \mathrm{L}$ glucose, which was a marked improvement from the $0.94 \mathrm{~g} / \mathrm{L}$ obtained in $\gamma$-PGA medium. Our previous work demonstrated that $B$. licheniformis CGMCC2876 secreted 89\% polysaccharides when glucose was supplied as the only carbon source [13, 21]. Conversely, when 5 or $10 \mathrm{~g} / \mathrm{L}$ glucose was added to the $\gamma$-PGA medium, the $\gamma$-PGA concentration decreased by 31.54 and $61.62 \%$, respectively (Fig. 2a; Table 1 ). Goto's study also showed that B. subtilis IF03335 produced $\gamma$-PGA with polysaccharides as by-products when glucose was supplied as a co-carbon source [22]. These results suggest that glucose activates the polysaccharide synthesis pathway, which then contributes to the increase in polysaccharides secretion.

\section{Effect of glucose on the molecular mass of the fermentation products}

The molecular masses of the fermentation products are shown in Fig. $2 \mathrm{~b}$. In the $\gamma$-PGA medium, $\gamma$-PGA was detected with an average mass ranging from $1.38 \times 10^{6}$ to $2.04 \times 10^{7} \mathrm{Da}$. With the addition of glucose at $5 \mathrm{~g} / \mathrm{L}$, the average masses were calculated to be $4.25 \times 10^{4}$, $4.57 \times 10^{4}, 5.38 \times 10^{4}$ and $6.38 \times 10^{4}$ Da. However,

(See figure on next Page.)

Fig. 1 B. licheniformis growth curve in the three media. a The growth curve, glycerol consumption and flocculant activity of the fermentation culture in pure $\gamma$-PGA medium. b The growth curve, glycerol and glucose consumption and flocculant activity of the fermentation culture in $\gamma$-PGA medium containing $5 \mathrm{~g} / \mathrm{L}$ glucose. $\mathbf{c}$ The growth curve, glycerol and glucose consumption and flocculant activity of the fermentation culture in $\gamma$-PGA medium containing $10 \mathrm{~g} / \mathrm{L}$ glucose 

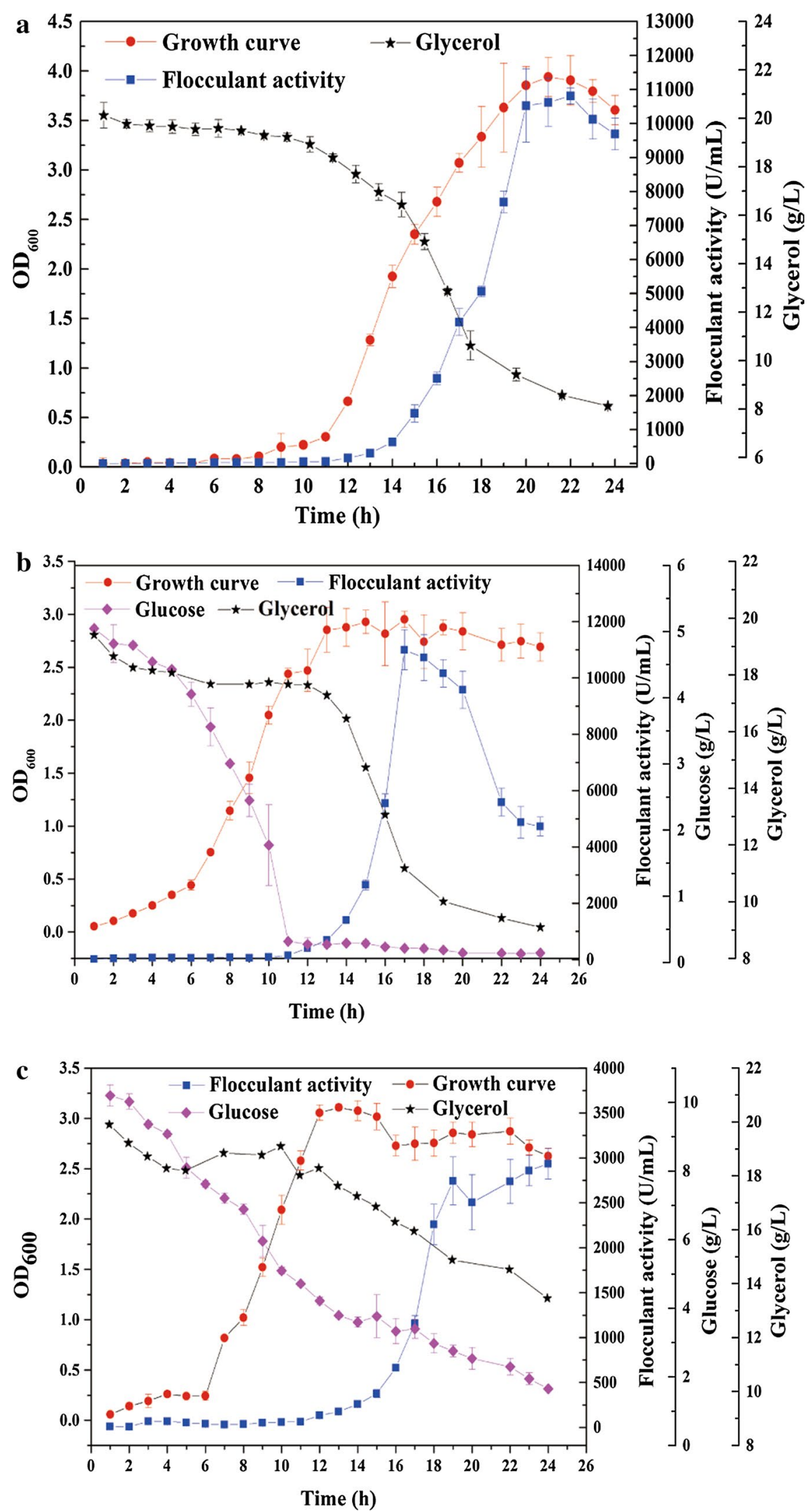
Table 1 The production and range of molecular mass of the fermentation products

\begin{tabular}{lllllrl}
\hline Sample & Crude extract (g/L) & Y-PGA (g/L) & Polysaccharide (g/L) & Y-PGA (\%) & Polysaccharide (\%) & Mw (Da) \\
\hline Y-PGA & $17.988 \pm 1.24$ & $14.82 \pm 1.69$ & $0.94 \pm 0.25$ & $82.41 \pm 7.39$ & $5.2 \pm 0.55$ & $1.38 \times 10^{6} \sim 2.04 \times 10^{7}$ \\
Y-PGA + 5 g & $19.968 \pm 1.57$ & $11.27 \pm 1.16$ & $0.89 \pm 0.46$ & $56.42 \pm 3.18$ & $4.45 \pm 0.79$ & $4.25 \times 10^{4} ; 4.57 \times 10^{4}$ \\
& & & & & & $5.38 \times 10^{4} ; 6.38 \times 10^{5}$ \\
Y-PGA + 10 g & $19.44 \pm 1.03$ & $6.15 \pm 0.85$ & $8.45 \pm 1.02$ & $31.63 \pm 4.33$ & $43.47 \pm 6.81$ & $2.57 \times 10^{4} ; 3.36 \times 10^{4}$ \\
& & & & & & $5.39 \times 10^{5} \sim 1.58 \times 10^{6}$
\end{tabular}
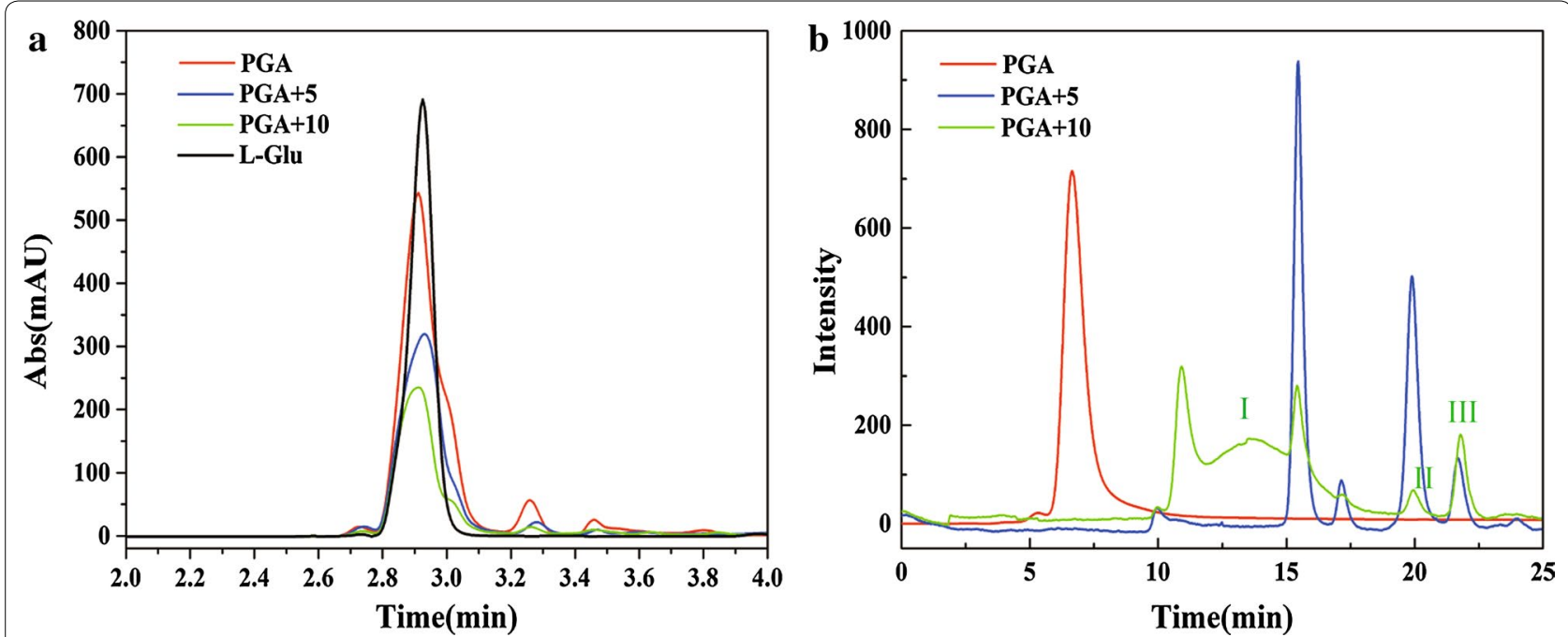

Fig. 2 The HPLC and GPC spectra of the B. licheniformis fermentation products. a The HPLC spectra of the B. licheniformis hydrolyzed fermentation products under three culture media. $\mathbf{b}$ The GPC traces of the purified fermentation product in the different culture media

with the addition of glucose at $10 \mathrm{~g} / \mathrm{L}$, broad molecular mass distributions were observed in peak I, ranging from $5.39 \times 10^{5}$ to $1.58 \times 10^{6} \mathrm{Da}$, whereas peaks II and III showed relatively low molecular masses of $2.57 \times 10^{4}$ and $3.36 \times 10^{4} \mathrm{Da}$, respectively (Table 1 ). The $\gamma$-PGA hydrolase PgdS was directly responsible for $\gamma$-PGA degradation to regulate the molecular mass [23]. Further results showed that pgdS mRNA expression was up-regulated in the medium containing glucose, indicating that the addition of glucose enhanced the expression of $\gamma$-PGA hydrolase PgdS to decrease the molecular mass of $\gamma$-PGA (Additional file 1).

The flocculating activity of bioflocculants is closely related to both their constituents and their molecular mass [24]. Under normal circumstances, $\gamma$-PGA shows higher flocculating activity than polysaccharides at the same concentration, and the flocculating activity increases with increasing of molecular mass. In the $\gamma$-PGA medium supplemented with glucose at $10 \mathrm{~g} / \mathrm{L}$, the flocculating activity of the culture was decreased by $70 \%$ due to the $43.47 \%$ decrease in $\gamma$-PGA concentration and to the presence of smaller molecules than those secreted from $\gamma$-PGA medium (Fig. 1).
Effect of glucose on the $B$. licheniformis metabolic pathway SWATH acquisition LC-MS/MS was employed to analyze the proteomes of $B$. licheniformis CGMCC2876 cultivated in three different media. In total, 969 intracellular proteins were detected (Additional file 2). The major proteins were involved in the EMP pathway, glycerol metabolism, and TCA cycle. Expression levels of proteins involved in the $\gamma$-PGA and polysaccharides biosynthesis are summarized and illustrated in the heat map in Fig. 3a.

\section{Carbon metabolism}

The activities of several enzymes involved in glycolysis increased with the addition of glucose compared with $\gamma$-PGA medium (Fig. 3a). However, the levels of these proteins decreased in the cell proteome when glucose was exhausted. Similar proteomic results were observed for B. licheniformis DSM 13, indicating that the expression of glycolytic proteins decreased under glucose-limiting conditions [25]. Some microbes can utilize the EMP pathway to synthesize glutamic acid as the precursor for $\gamma$-PGA synthesis $[26,27]$. Similar to the findings reported in B. subtilis NX-2 [7], the glycolytic pathway substrates 


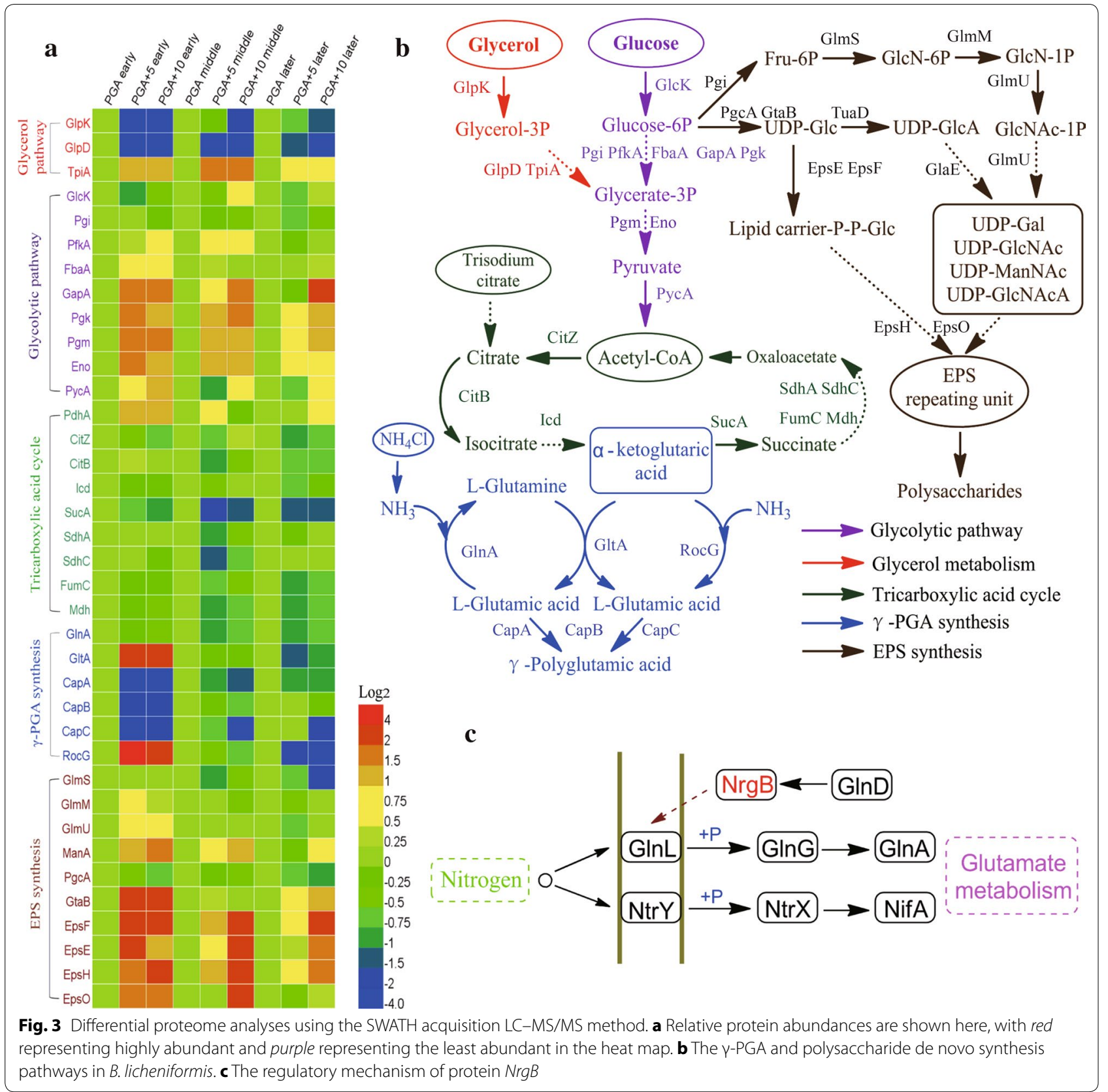

were supplied for cell growth, and the monomers were provided for polysaccharide biosynthesis (Fig. 3b).

As illustrated in Fig. 3a, glycerol metabolism was markedly inhibited by the addition of glucose, while glycerol was used as a preferred carbon source in $\gamma$-PGA synthesis, which was consistent with the reports of some other studies $[6,15,28,29]$.

TCA cycle is an essential part of $\gamma$-PGA synthetic pathways and produces a precursor ( $\alpha$-ketoglutaric acid) for glutamate synthesis $[22,30]$. However, a large amount of pyruvic acid from glycolysis is degraded via the Krebs cycle. In the medium containing glucose at $5 \mathrm{~g} / \mathrm{L}$, the levels of SucA and SdhC decreased by 3.59- and 2.01fold, respectively (Additional file 3 ) after the glucose was exhausted. The same results revealed that the decrease in $S u c A$ and $S d h C$ was beneficial for both the accumulation of $\alpha$-ketoglutaric acid and the increase in glutamate for producing $\gamma$-PGA [31]. Thus, glycerol metabolism was changed to glycolysis when glucose was added to the medium as a co-carbon source.

\section{$\gamma$-PGA biosynthesis}

We observed that the $\gamma$-PGA synthetic enzyme system $(C a p A B C)$ markedly decreased during the exponential 
growth phase $\left(\mathrm{OD}_{600}=1.0\right)$ when glucose was added (Fig. 3a). Tannler et al. reported that glucose repressed a series of secondary catabolic proteins via carbon control protein $\mathrm{N}(C c p N)$ [32]. The cap operon might be regulated by $C c p N$. In the medium containing glucose at $5 \mathrm{~g} / \mathrm{L}, \operatorname{Cap} A B C$ content in the cells mildly increased as glucose was exhausted, then returned to the same level as that in the cells cultured in $\gamma$-PGA medium. However, in the $10 \mathrm{~g} / \mathrm{L}$ glucose medium, CapABC was repressed during the entire fermentation process.

Interestingly, GltA and RocG, which are both involved in $\gamma$-PGA biosynthesis, were up-regulated with the addition of glucose. GltA is a major regulatory link between carbon and amino acid metabolism. The lack of the gltAB operon limits B. subtilis growth on glucose/ammonium media [33]. However, RocG catalyzes the reaction (glutamate $+\mathrm{NAD}^{+} \rightarrow \alpha$-ketoglutarate $\left.+\mathrm{NH}_{3}+\mathrm{NADH}\right)$, which provides rapidly metabolizable carbon- or nitrogen-containing compounds for biosynthesis [34].

\section{EPS biosynthesis}

Several intracellular enzymes (GlmS, GlmM, GlmU, EpsE, EpsF, EpsH, EpsO, ManA, PgcA and GtaB) that participate in polysaccharide synthesis [35] were more abundant in the medium containing $10 \mathrm{~g} / \mathrm{L}$ glucose than in $\gamma$-PGA medium (Fig. 3a). The expression of the epsA$O$ operon was maintained at a high level during polysaccharide synthesis. The bacterial cells were stimulated to activate a series of operons to synthesize polysaccharides, resulting in the diversion of the carbon flux from $\gamma$-PGA synthesis to polysaccharides.

\section{Stress response proteins}

Several regulatory proteins related to carbon and nitrogen metabolism are shown in Table 2. The addition of glucose resulted in an increase in $C c p A$ during the exponential growth phase $\left(\mathrm{OD}_{600}=1.0\right)$. CcpA (carbon catabolite control A) is a central regulator for coordinating the carbon metabolism and energy sources to maximize efficiency via carbon catabolite repression (CCR) and carbon catabolite activation (CCA) [36]. CcpA has also been reported to repress TCA cycle and activate the EMP pathway in response to the presence of glucose [37].
However, CcpA expression remained high when $5 \mathrm{~g} / \mathrm{L}$ glucose was exhausted. Belitsky's study showed that glutamic acid synthesis was accelerated by $C c p A$ through the activation of GltAB and the repression of RocG [34]. Conversely, the deletion of $c c p A$ repressed icaADBC transcription and inhibited polysaccharide formation in S. epidermidis [38]. Similarly, an S. gordonii ccpA mutant showed severe impairment of extracellular polysaccharide production [39].

As shown in Table 2, $C c p N$ increased sharply in the early growth phase when glucose was added. When glucose was exhausted, $C c p N$ decreased to the level observed in the cells in $\gamma$-PGA medium. $C c p N$ in $B$. subtilis has been characterized as a repressor of two gluconeogenic genes (gapB/pckA, and $g l p F K / g l p D)$ that are involved in glycerol metabolism [32, 36]. When the glucose were consumed in the $5 \mathrm{~g} / \mathrm{L}$ glucose medium, $C c p N$ decreased to the level detected in the bacterial cells cultured in $\gamma$-PGA medium. $C c p N$ remarkably altered the distribution of carbon fluxes in $B$. licheniformis CGMCC2876 by rerouting the main carbon fluxes from glycerol metabolism to glycolysis.

As shown in Fig. 3c, $\mathrm{NrgB}$ relayed information on the ammonium availability to downstream regulatory factors and activated $G \ln A$ and $G \ln G$, which are involved in glutamate metabolism [40]. $\mathrm{NrgB}$ was strongly repressed during the exponential growth phase when glucose was added. Concomitant with the fermentation process, $\mathrm{NrgB}$ content in the proteome of the cells in the medium supplemented with $5 \mathrm{~g} / \mathrm{L}$ glucose increased to the same level as that detected in the cells in $\gamma$-PGA medium, whereas $\mathrm{NrgB}$ levels were always low in the medium containing $10 \mathrm{~g} / \mathrm{L}$ glucose. However, glutamine is an optimal nitrogen source for $B$. licheniformis growth. When glutamine was exhausted, alternative nitrogen sources such as ammonium were utilized [41]. In $\gamma$-PGA medium, $\operatorname{NrgB}$ facilitated ammonium utilization and activated $G \ln A$ and $G \ln G$ to promote glutamine synthesis, which was beneficial for $\gamma$-PGA synthesis. When glucose was added to the medium, the down-regulation of $\mathrm{NrgB}$ resulted in the use of glutamine as a nitrogen source and promoted cell growth. These results suggested that $N r g B$ was a positive regulator of ammonium utilization

Table 2 Proteomics changes in related regulatory proteins

\begin{tabular}{|c|c|c|c|c|c|c|c|c|c|}
\hline Protein & PGA early & PGA+5 early & PGA+10 early & PGA middle & PGA+5 middle & PGA+10 middle & PGA late & PGA+5 late & PGA+10 late \\
\hline NadR & 1 & 5.93 & 4.804 & 1 & 1.12 & 1.46 & 1 & 0.86 & 0.94 \\
\hline NrgB & 1 & 0.32 & 0.273 & 1 & 1.22 & 0.63 & 1 & 1.17 & 0.69 \\
\hline CcpA & 1 & 1.64 & 1.92 & 1 & 2.13 & 2.34 & 1 & 0.76 & 0.55 \\
\hline CcpN & 1 & 3.97 & 6.16 & 1 & 1.89 & 3.04 & 1 & 0.58 & 0.42 \\
\hline CodY & 1 & 0.41 & 0.27 & 1 & 1.22 & 1.05 & 1 & 0.42 & 0.52 \\
\hline
\end{tabular}


and rerouted the main nitrogen flux from glutamine to ammonium.

\section{qPCR analysis}

Nine selected genes were analyzed in detail via quantitative PCR (Table 3). These genes were selected based on their osculating roles in polysaccharide and $\gamma$-PGA synthesis with a wide range of abundances. The majority of these selected genes exhibited good correlations between the changes in mRNA expression levels and the corresponding protein abundance. Glck and fruK, which were involved in glycolysis and controlled by $C c p A$, had a decreased expression level with the addition of glucose. Glpk mRNA expression, which was repressed by $C c p N$, was significantly down-regulated with the addition of glucose. $G \ln A$, which catalyzes the conversion of $\alpha$-ketoglutarate and $\mathrm{NH}_{3}$ to glutamate, was mildly downregulated at the proteome level in the presence of glucose. However, $g \ln A$ mRNA expression was up-regulated in the cells cultured in the medium containing glucose. The $\operatorname{gln} A$ gene (glutamine synthetase) has been reported to be regulated by small regulatory RNAs (sRNAs) that base-paired with the mRNA to alter mRNA stability and translation initiation in a Bacillus strain [42, 43]. These results confirmed the accuracy of the proteome quantification and suggested that the regulatory processes associated with the $g \ln A$ gene predominantly occurred at the level of post-transcriptional regulation.

Models for the carbon and nitrogen metabolic flux regulation with the effects of glucose in $B$. licheniformis CGMCC2876 are proposed in Fig. 4. When the $\gamma$-PGA medium was supplemented with glucose, the expression of the regulatory proteins $(C c p A$ and $C c p N$ for carbon regulation and $\operatorname{NrgB}$ for nitrogen regulation) was initiated. During the early stage of the fermentation process, $C c p A$ and $C c p N$ co-enhanced the glycolysis intensity and suppressed carbon flux into TCA cycle to accelerate the bacterial growth. In contrast, $C c p N$ cut off the carbon flux from glycerol metabolism, further reducing $\gamma$-PGA production. CcpA activated a series of operons ( $\mathrm{glm}$ and eps $A-O)$ to reallocate the carbon flux and produce polysaccharide. When glucose in the medium was exhausted, the down-regulation of $C c p N$ resulted in the accelerated utilization of glycerol. $C c p A$ increased glutamic acid synthesis through the activation of GltAB and the repression of RocG. These changes were beneficial for $\gamma$-PGA biosynthesis. For the regulation of nitrogen metabolic flux, the down-regulation of $\mathrm{NrgB}$ switched the major source of nitrogen from $\mathrm{NH}_{4}^{+}$to glutamate. When glucose in the $5 \mathrm{~g} / \mathrm{L}$ glucose medium was exhausted, $C c p N$ expression gradually decreased, and the carbon flux from glycerol metabolism was regained. Simultaneously, when $\operatorname{NrgB}$ was up-regulated at the proteome level, the ability of the microbe to use $\mathrm{NH}_{4}^{+}$as its primary nitrogen source was restored, and the ability of glutamate synthesis to secrete $\gamma$-PGA was enhanced.

\section{Conclusion}

In this study, we demonstrated the effect of glucose on fermentation products through qualitative and quantitative analyses for the first time. The $\gamma$-PGA concentration decreased by 31.54 and $61.62 \%$ when the culture medium was supplemented with glucose at 5 and $10 \mathrm{~g} / \mathrm{L}$, respectively. However, the polysaccharide concentration rose sharply from 5.2 to $43.47 \%$ with the addition of glucose at $10 \mathrm{~g} / \mathrm{L}$. The SWATH-MS method was used to clarify bacterial metabolic regulation at the protein level, resulting in the detection of 969 intracellular proteins. Carbon control proteins $(C c p A$ and $C c p N)$ redistributed the carbon flux from $\gamma$-PGA to polysaccharide production in the presence of glucose. Simultaneously, the regulatory protein $N r g B$ converted the major nitrogen metabolic flux from $\mathrm{NH}_{4}{ }^{+}$to glutamate. Overall, the central metabolic turnover processes of two different extracellular polymeric substances in B. licheniformis CGMCC2876 were elucidated and provided an effective fermentation strategy for regulating the production of polysaccharides and $\gamma$-PGA. Our results offer different molecular weights for

Table 3 Transcriptomic changes in selected genes

\begin{tabular}{llllllllll}
\hline Gene & PGA early & PGA+5 early & PGA+10 early & PGA middle & PGA+5 middle & PGA+10 middle & PGA late & PGA+5 late & PGA+10 late \\
\hline glcK & $1.00 \pm 0.13$ & $1.28 \pm 0.11$ & $3.36 \pm 0.07$ & $0.41 \pm 0.06$ & $0.06 \pm 0.01$ & $2.09 \pm 0.23$ & $0.10 \pm 0.04$ & $0.04 \pm 0.01$ & $1.09 \pm 0.17$ \\
fruK & $1.00 \pm 0.21$ & $2.46 \pm 0.19$ & $4.68 \pm 0.78$ & $1.02 \pm 0.15$ & $1.27 \pm 0.28$ & $2.02 \pm 0.37$ & $1.69 \pm 0.21$ & $0.92 \pm 0.11$ & $1.93 \pm 0.40$ \\
glpK & $1.00 \pm 0.17$ & $0.42 \pm 0.05$ & $0.08 \pm 0.004$ & $0.16 \pm 0.03$ & $0.86 \pm 0.21$ & $0.10 \pm 0.02$ & $0.21 \pm 0.05$ & $0.28 \pm 0.03$ & $0.12 \pm 0.01$ \\
icd & $1.00 \pm 0.18$ & $0.52 \pm 0.09$ & $0.48 \pm 0.07$ & $0.11 \pm 0.03$ & $0.07 \pm 0.01$ & $0.29 \pm 0.04$ & $0.05 \pm 0.007$ & $0.04 \pm 0.01$ & $0.03 \pm 0.005$ \\
glnA & $1.00 \pm 0.19$ & $1.66 \pm 0.23$ & $1.17 \pm 0.19$ & $0.67 \pm 0.08$ & $0.46 \pm 0.11$ & $0.99 \pm 0.24$ & $0.89 \pm 0.17$ & $0.33 \pm 0.05$ & $0.44 \pm 0.08$ \\
suCA & $1.00 \pm 0.09$ & $1.06 \pm 0.04$ & $0.83 \pm 0.39$ & $5.26 \pm 0.57$ & $2.59 \pm 0.31$ & $3.78 \pm 0.86$ & $0.36 \pm 0.06$ & $2.87 \pm 0.33$ & $2.71 \pm 0.37$ \\
pgsAA & $1.00 \pm 0.12$ & $0.24 \pm 0.07$ & $0.17 \pm 0.02$ & $0.37 \pm 0.08$ & $0.46 \pm 0.09$ & $0.15 \pm 0.01$ & $0.06 \pm 0.007$ & $0.07 \pm 0.004$ & $0.29 \pm 0.03$ \\
pgsB & $1.00 \pm 0.07$ & $0.13 \pm 0.006$ & $0.17 \pm 0.02$ & $0.14 \pm 0.03$ & $0.56 \pm 0.02$ & $0.05 \pm 0.003$ & $0.02 \pm 0.001$ & $0.03 \pm 0.007$ & $0.09 \pm 0.01$ \\
pgsC & $1.00 \pm 0.07$ & $0.29 \pm 0.03$ & $0.23 \pm 0.01$ & $0.03 \pm 0.001$ & $0.65 \pm 0.04$ & $0.11 \pm 0.01$ & $0.02 \pm 0.005$ & $0.01 \pm 0.001$ & $0.01 \pm 0.002$ \\
\hline
\end{tabular}




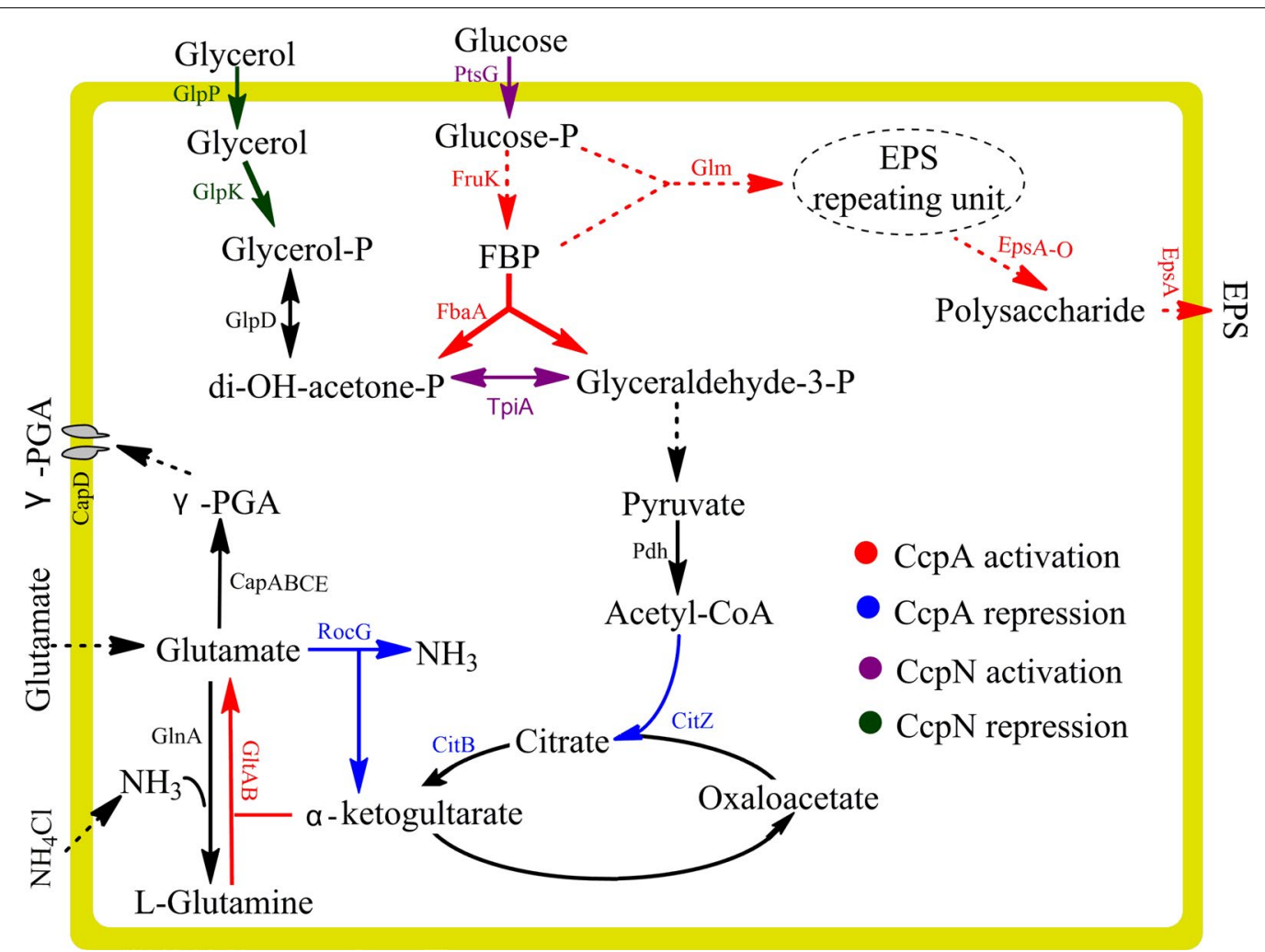

Fig. 4 Proposed models for the central metabolic turnover process by which B. licheniformis produces $Y$-PGA and polysaccharide

diverse applications through the regulation of the ratio of the carbon source in the culture. Moreover, the regulatory mechanism provides meaningful biological information for the metabolic engineering of B. licheniformis for enhanced flocculate production.

\section{Methods}

\section{Strain and media}

The B. licheniformis CGMCC2876 used in this study was isolated by our laboratory [13].

The pre-culture medium consisted of the following components (g/L): glucose, 10; urea, $0.5 ; \mathrm{MgSO}_{4}, 0.2$; $\mathrm{KH}_{2} \mathrm{PO}_{4}, 0.1 ; \mathrm{K}_{2} \mathrm{HPO}_{4}, 0.1 ; \mathrm{NaCl}, 0.1$ and yeast extract, 0.5 (pH 7.2). The $\gamma$-PGA production medium contained the following components $(\mathrm{g} / \mathrm{L})$ : trisodium citrate, 20 ; glycerol, 20; $\mathrm{NH}_{4} \mathrm{Cl}$, 9; sodium glutamate, $10 ; \mathrm{MgSO}_{4}, 0.5$; and $\mathrm{K}_{2} \mathrm{HPO}_{4}, 0.5$ (pH 7.2). A total of 5 or $10 \mathrm{~g} / \mathrm{L}$ of glucose was added to the $\gamma$-PGA production medium.

The cells were first maintained in an Erlenmeyer flask containing pre-culture medium at $37^{\circ} \mathrm{C}$ at $200 \mathrm{rpm}$ for $17 \mathrm{~h}$ and then transferred (at a $4 \%$ inoculum ratio) into a $250 \mathrm{~mL}$ Erlenmeyer flask with $50 \mathrm{~mL}$ of $\gamma$-PGA production medium (containing $5,10 \mathrm{~g} / \mathrm{L}$, or no glucose) for $\gamma$-PGA production.

\section{SWATH acquisition LC-MS/MS method analysis}

For the preparation of cytoplasm proteins, the bacteria were harvested by centrifugation $(12,000 \times g, 15 \mathrm{~min}$, $4{ }^{\circ} \mathrm{C}$ ) at three sampling times (early, middle and late). The early sampling time was during exponential growth, when $\mathrm{OD}_{600}=1.0$. The middle sampling time was $1 \mathrm{~h}$ after glucose was exhausted in the $\gamma$-PGA production medium supplemented with $5 \mathrm{~g} / \mathrm{L}$ glucose. The late sampling time was at the end of fermentation. The pellets were washed with TE buffer $(10 \mathrm{mM}$ Tris and $1 \mathrm{mM}$ EDTA, pH 7.5) and then resuspended in TE buffer [44]. The resuspended cells were disrupted twice at $25 \mathrm{kpsi}$ at $4{ }^{\circ} \mathrm{C}$ using a homogenizer (One Shot Model, Constant Systems, UK). The cell debris was removed by centrifugation at $14,000 \times g$ for $15 \mathrm{~min}$ at $4{ }^{\circ} \mathrm{C}$. SWATH acquisition LC-MS/MS was performed using an Eksigent nanoLCultra system coupled with a Triple-TOF5600 Mass Spectrometer (ABSCIEX, Canada). Details of the parameters and data analysis for SWATH were reported by Yu et al. [31].

\section{qPCR analysis}

Concomitant with the protein extraction, RNA was immediately extracted from the samples during 
exponential growth $\left(\mathrm{OD}_{600}=1.0\right), 1 \mathrm{~h}$ after glucose was exhausted in the $\gamma$-PGA production medium supplemented with $5 \mathrm{~g} / \mathrm{L}$ glucose, and at the end of fermentation using the MiniBEST Universal RNA Extraction Kit (TaKaRa, Japan). The isolated RNA was quantified using a Spectrophotometer Q6000 (Quawell, USA). A high capacity cDNA reverse transcription kit (Applied Biosystems, USA) and a TransStart Top Green qPCR SuperMix Kit (TransGen Biotech, China) were used for reverse transcription and real-time PCR, respectively. The realtime PCR analysis was performed using a StepOne RealTime PCR System (Applied Biosystems, USA). Reactions without the CDNA template were used as the negative controls, and $\gamma$-PGA medium without glucose was used as the reference in the calculations.

\section{Purification of fermentation products}

After $24 \mathrm{~h}$ of fermentation, the culture broths were centrifuged at $10,000 \times g$ for $15 \mathrm{~min}$ to remove the cells. Three volumes of ethanol were added to the supernatant to precipitate the crude products. Then, the crude products were dissolved using distilled water and dialyzed (molecular weight cut-off of $7000 \mathrm{Da}$ ) in distilled water overnight. Finally, the sample was lyophilized to obtain the purified products [45].

\section{Qualitative and quantitative analyses of the fermentation products}

The total sugar content of the purified products was determined by the phenol-sulfuric acid method using glucose as the standard solution [46]. The total protein content was measured by the Bradford method using a protein assay kit (Bio-Rad, USA).

To measure the $\gamma$-PGA content, the purified products were dissolved in $6 \mathrm{M} \mathrm{HCl}$ to hydrolyze the $\gamma$-PGA. The mixtures were maintained at $110^{\circ} \mathrm{C}$ for $12 \mathrm{~h}$. The hydrolysates were neutralized and metered volumetrically and then characterized by HPLC for qualitative and quantitative analysis. The HPLC analysis was performed on an Agilent $1200 \mathrm{HPLC}$ system using an Agilent $\mathrm{HC}-\mathrm{C}_{18}$ $(25 \mathrm{~cm} \times 4.6 \mathrm{~mm})$ column and a UV detector $(210 \mathrm{~nm})$. The mobile phase consisted of $10 \mathrm{mM} \mathrm{KH} \mathrm{PO}_{4}$ (pH 2.5) and methanol $(5 \%, \mathrm{v} / \mathrm{v})$ at a flow rate of $0.5 \mathrm{~mL} / \mathrm{min}$. Pure sodium glutamate was used as the standard compound [47].

\section{Determination of the molecular masses of the fermentation products}

Molecular mass was evaluated by high-performance gel permeation chromatography (HPGPC) coupled with refractive index (RI) detection using a TSK G4000PWxl column (Tosoh, Japan). The mobile phase was $\mathrm{NaN}_{3}$ $(0.01 \%)$ at a flow rate of $0.5 \mathrm{~mL} / \mathrm{min}$. The column temperature and pressure were maintained at $30{ }^{\circ} \mathrm{C}$ and 1.3 $\mathrm{MPa}$, respectively. A dextran $\mathrm{T}$ series (Pharmacia, Sweden) was used as the standard compound for the molecular mass determination [48].

\section{Additional files}

Additional file 1. qPCR analysis of pgdS gene expression in $B$ licheniformis.

Additional file 2. Raw data of SWATH acquisition LC-MS/MS.

Additional file 3. Date used in Fig. 3a.

\section{Abbreviations}

EPS: extracellular polysaccharides; $\gamma$-PGA: poly-gamma-glutamic acid; SWATH: sequential window acquisition of all theoretical fragment-ion spectra; EMP: Embden-Meyerhof-Parnas pathway; TCA: tricarboxylic acid cycle.

\section{Authors' contributions}

WCY, ZC, NH, CJZ, YPW and QBL designed the experiments. WCY, ZC and HY preformed the experiments. WCY, ZC and NH analyzed the results. WCY, ZC, $\mathrm{NH}$ and SY wrote the manuscript which was reviewed and approved by all authors. All authors read and approved the final manuscript.

\section{Author details}

${ }^{1}$ Department of Chemical and Biochemical Engineering, College of Chemistry and Chemical Engineering, Xiamen University, Xiamen 361005, People's Republic of China. ${ }^{2}$ The Key Lab for Synthetic Biotechnology of Xiamen City, Xiamen University, Xiamen 361005, People's Republic of China. ${ }^{3}$ Fujian Provincial Key Laboratory of Fire Retardant Materials, College of Materials, Xiamen University, Xiamen 361005, People's Republic of China. ${ }^{4}$ Department of Chemistry, State University of New York at Binghamton, Binghamton, NY 13902, USA.

\section{Acknowledgements}

This work was financially supported by the National Key Research and Development Plan (2016YFD0400205), National Natural Science Foundation of China (51378444, 21676221), the University of Science and Technology in Fujian Province in the cooperative major project $(2015 \mathrm{H} 6004)$ and Xiamen Southern Oceanographic Center (15GYY024NF03). We also gratefully acknowledge the Analysis and Testing Center, School of Life Sciences, Xiamen University and the Fujian Provincial Scientific and Technological Innovation Platform of China (2014H2006) for their technical support and AJE for language polishing work.

Competing interests

The authors declare that they have no competing interests.

Received: 6 September 2016 Accepted: 28 January 2017

Published online: 08 February 2017

\section{References}

1. Shih $I-L$, Van $Y-T$. The production of poly-( $Y$-glutamic acid) from microorganisms and its various applications. Bioresour Technol. 2001;79:207-25

2. Bajaj I, Singhal R. Poly (glutamic acid)-an emerging biopolymer of commercial interest. Bioresour Technol. 2011;102:5551-61.

3. Ndikubwimana T, Zeng XH, Liu Y, Chang JS, Lu YH. Harvesting of microalgae Desmodesmus sp. F51 by bioflocculation with bacterial bioflocculant. Algal Res. 2014;6:186-93.

4. Hajdu I, Bodnár M, Csikós Z, Wei S, Daróczi L, Kovács B, Győri Z, Tamás J, Borbély J. Combined nano-membrane technology for removal of lead ions. J Membr Sci. 2012;409-410:44-53.

5. Ashiuchi M. Microbial production and chemical transformation of polygamma-glutamate. Microb Biotechnol. 2013;6:664-74. 
6. Ko YH, Gross RA. Effects of glucose and glycerol on gamma-poly(glutamic acid) formation by Bacillus licheniformis ATCC 9945a. Biotechnol Bioeng. 1998:57:430-7.

7. Yao J, Xu H, Shi N, Cao X, Feng X, Li S, Ouyang P. Analysis of carbon metabolism and improvement of $\gamma$-polyglutamic acid production from Bacillus subtilis NX-2. Appl Biochem Biotechnol. 2009;160:2332-41.

8. Zhou T, Schneider KE, Li XZ. Development of biocontrol agents from food microbial isolates for controlling post-harvest peach brown rot caused by Monilinia fructicola. Int J Food Microbiol. 2008;126:180-5.

9. MsadekT, Kunst F, Klier A, Rapoport G. DegS-DegU and ComP-ComA modulator-effector pairs control expression of the Bacillus subtilis pleiotropic regulatory gene degQ. J Bacteriol. 1991;173:2366-77.

10. Stanley NR, Lazazzera BA. Defining the genetic differences between wild and domestic strains of Bacillus subtilis that affect poly-gammadl-glutamic acid production and biofilm formation. Mol Microbiol. 2005:57:1143-58.

11. Liu J, He D, Li XZ, Gao S, Wu H, Liu W, Gao X, Zhou T. Gamma-polyglutamic acid (gamma-PGA) produced by Bacillus amyloliquefaciens C06 promoting its colonization on fruit surface. Int J Food Microbiol. 2010;142:190-7.

12. Feng J, Gu Y, Sun Y, Han L, Yang C, Zhang W, Cao M, Song C, Gao W, Wang S. Metabolic engineering of Bacillus amyloliquefaciens for polygamma-glutamic acid (gamma-PGA) overproduction. Microb Biotechnol. 2014;7:446-55.

13. Xiong Y, Wang Y, Yu Y, Li Q, Wang H, Chen R, He N. Production and characterization of a novel bioflocculant from Bacillus licheniformis. Appl Environ Microbiol. 2010;76:2778-82.

14. Chen Z, Liu P, Li Z, Yu W, Wang Z, Yao H, Wang Y, Li Q, Deng X, He N. Identification of key genes involved in polysaccharide bioflocculant synthesis in Bacillus licheniformis. Biotechnol Bioeng. 2017;1 14:645-55.

15. Yan $\mathrm{S}$, Yao H, Chen Z, Zeng S, Xi X, Wang Y, Li Q, He N. Poly- $\gamma$-glutamic acid produced from Bacillus licheniformis CGMCC 2876 as a potential substitute for polyacrylamide in the sugarcane industry. Biotechnol Prog. 2015;31:1287-94.

16. Salehizadeh H, Shojaosadati SA. Extracellular biopolymeric flocculants: recent trends and biotechnological importance. Biotechnol Adv. 2001;19:371-85

17. Luo ZT, Guo Y, Liu JD, Qiu H, Zhao MM, Zou W, Li SB. Microbial synthesis of poly-gamma-glutamic acid: current progress, challenges, and future perspectives. Biotechnol Biofuels. 2016;9:134

18. Kunioka M. Biosynthesis of poly ( $\gamma$-glutamic acid) from L-glutamine, citric acid and ammonium sulfate in Bacillus subtilis IFO3335. Appl Microbiol Biotechnol. 1995;44:501-6.

19. Kongklom N, Luo HZ, Shi ZP, Pechyen C, Chisti Y, Sirisansaneeyakul S. Production of poly-gamma-glutamic acid by glutamic acid-independent Bacillus licheniformis TISTR 1010 using different feeding strategies. Biochem Eng J. 2015;100:67-75.

20. Mathiazhakan K, Ayed D, Tyagi RD. Kinetics of lipid production at lab scale fermenters by a new isolate of Yarrowia lipolytica SKY7. Bioresour Technol. 2016;221:234-40

21. Zhuang XL, Wang YP, Li QB, Yan S, He N. The production of bioflocculants by Bacillus licheniformis using molasses and its application in the sugarcane industry. Biotechnol Bioproc E. 2012:17:1041-7.

22. Goto A, Kunioka M. Biosynthesis and Hydrolysis of Poly( $($-glutamic acid) from Bacillus subtilis IF03335. Biosci Biotechnol Biochem. 2014:56:1031-5.

23. Feng J, Gao W, Gu Y, Zhang W, Cao M, Song C, Zhang P, Sun M, Yang C, Wang S. Functions of poly-gamma-glutamic acid ( $\gamma-P G A)$ degradation genes in $\gamma-P G A$ synthesis and cell morphology maintenance. Appl Microbiold Biotechnol. 2014;98:6397-407.

24. Salehizadeh $\mathrm{H}$, Yan N. Recent advances in extracellular biopolymer flocculants. Biotechnol Adv. 2014;32:1506-22.

25. Voigt B, le Hoi T, Jurgen B, Albrecht D, Ehrenreich A, Veith B, Evers S, Maurer KH, Hecker M, Schweder T. The glucose and nitrogen starvation response of Bacillus licheniformis. Proteomics. 2007;7:413-23.

26. Cheng C, Asada Y, Aida T. Production of gamma-polyglutamic acid by Bacillus licheniformis A35 under denitrifying conditions. Agric Biol Chem. 1989;53:2369-75

27. Ito Y, Tanaka T, Ohmachi T, Asada Y. Glutamic acid independent production of poly(Y-glutamic acid) by Bacillus subtilis TAM-4. Biosci Biotechnol Biochem. 2014;60:1239-42.
28. Hoppensack A, Oppermann-Sanio FB, Steinbuchel A. Conversion of the nitrogen content in liquid manure into biomass and polyglutamic acid by a newly isolated strain of Bacillus licheniformis. FEMS Microbiol Lett. 2003:218:39-45.

29. Shih IL, Van YT, Yeh LC, Lin HG, Chang YN. Prodiction of a biopolymer flocculant from Bacillus licheniformis and its flocculation properties. Bioresour Technol. 2001;78:267-72.

30. Cromwick A-M, Gross RA. Investigation by NMR of metabolic routes to bacterial y-poly(glutamic acid) using ${ }^{13} \mathrm{C}$-labeled citrate and glutamate as media carbon sources. Can J Microbiol. 1995;41:902-9.

31. Yu W, Chen Z, Shen L, Wang Y, Li Q, Yan S, Zhong CJ, He N. Proteomic profiling of Bacillus licheniformis reveals a stress response mechanism in the synthesis of extracellular polymeric flocculants. Biotechnol Bioeng. 2016;113:797-806.

32. Tannler S, Fischer E, Le Coq D, Doan T, Jamet E, Sauer U, Aymerich S. CcpN controls central carbon fluxes in Bacillus subtilis. J Bacteriol. 2008; 190:6178-87.

33. Wacker I, Ludwig H, Reif I, Blencke HM, Detsch C, Stulke J. The regulatory link between carbon and nitrogen metabolism in Bacillus subtilis: regulation of the gltAB operon by the catabolite control protein CcpA. Microbiology. 2003;149:3001-9.

34. Belitsky BR, Kim HJ, Sonenshein AL. CcpA-dependent regulation of Bacillus subtilis glutamate dehydrogenase gene expression. J Bacteriol. 2004; 186:3392-8

35. Yan S, Wang N, Chen Z, Wang Y, He N, Peng Y, Li Q, Deng X. Genes encoding the production of extracellular polysaccharide bioflocculant are clustered on a 30-kb DNA segment in Bacillus licheniformis. Funct Integr Genom. 2013;13:425-34.

36. Fujita Y. Carbon catabolite control of the metabolic network in Bacillus subtilis. Biosci Biotechnol Biochem. 2009;73:245-59.

37. Moreno MS, Schneider BL, Maile RR, Weyler W, Saier MH. Catabolite repression mediated by the CcpA protein in Bacillus subtilis: novel modes of regulation revealed by whole-genome analyses. Mol Microbiol. 2004;39:1366-81

38. Sadykov MR, Hartmann T, Mattes TA, Hiatt M, Jann NJ, Zhu Y, Ledala N, Landmann R, Herrmann M, Rohde H, et al. CcpA coordinates central metabolism and biofilm formation in Staphylococcus epidermidis. Microbiology. 2011;157:3458-68.

39. Zheng L, Chen Z, Itzek A, Herzberg MC, Kreth J. CcpA regulates biofilm formation and competence in Streptococcus gordonii. Mol Oral Microbiol. 2012;27:83-94.

40. Detsch C, Stulke J. Ammonium utilization in Bacillus subtilis: transport and regulatory functions of NrgA and NrgB. Microbiology. 2003;149:3289-97.

41. Fisher SH, Brandenburg JL, Wray LV. Mutations in Bacillus subtilis glutamine synthetase that block its interaction with transcription factor TnrA. Mol Microbiol. 2002:45:627-35.

42. Feng J, Gu Y, Quan Y, Cao M, Gao W, Zhang W, Wang S, Yang C, Song C. Improved poly-gamma-glutamic acid production in Bacillus amyloliquefaciens by modular pathway engineering. Metab Eng. 2015;32:106-15.

43. Wang J, Rennie W, Liu C, Carmack CS, Prevost K, Caron MP, Masse E, Ding $Y$, Wade JT. Identification of bacterial sRNA regulatory targets using ribosome profiling. Nucleic Acids Res. 2015;43:10308-20.

44. Voigt B, Schweder T, Becher D, Ehrenreich A, Gottschalk G, Feesche J, Maurer KH, Hecker M. A proteomic view of cell physiology of Bacillus licheniformis. Proteomics. 2004:4:1465-90.

45. Do JH, Chang HN, Lee SY. Efficient recovery of $Y$-poly (glutamic acid) from highly viscous culture broth. Biotechnol Bioeng. 2001;76:219-23.

46. Cuesta G, Suarez N, Bessio Ml, Ferreira F, Massaldi H. Quantitative determination of pneumococcal capsular polysaccharide serotype 14 using a modification of phenol-sulfuric acid method. J Microbiol Methods. 2003;52:69-73.

47. Hezayen FF, Rehm BHA, Eberhardt R, Steinbuchel A. Polymer production by two newly isolated extremely halophilic archaea: application of a novel corrosion-resistant bioreactor. Appl Microbiol Biotechnol. 2000;54:319-25.

48. Zhao C, Zhang Y, Wei X, Hu Z, Zhu F, Xu L, Luo M, Liu H. Production of ultra-high molecular weight poly-gamma-glutamic acid with Bacillus licheniformis P-104 and characterization of its flocculation properties. Appl Biochem Biotechnol. 2013;170:562-72. 